\title{
Lung MRI as a Possible Alternative to CT Scan for Patients With Primary Immune Deficiencies and Increased Radiosensitivity
}

\author{
Goffredo Serra, MD; Cinzia Milito, MD; Milica Mitrevski, MD; Guido Granata, MD; \\ Helene Martini, MD; Anna Maria Pesce, MD; Ifigenia Sfika, MD; Livia Bonanni, MD; \\ Carlo Catalano, MD; Francesco Fraioli, MD; and Isabella Quinti, MD, PhD
}

\begin{abstract}
Background: Patients with common variable immunodeficiency (CVID) suffer from respiratory infections leading over time to permanent lung damage. Increased radiosensitivity has been described, and clinicians should consider a risk-benefit assessment when ordering a CT scan, in that the exact level of "safe" radiation exposure is unknown.

Methods: Twenty-one patients with CVID were evaluated with chest CT scan, MRI, and pulmonary function tests on the same day. MRI protocol included a T2-weighted rotating blade-like k-space covering sequence (time repetition, 2,000; echo train $=27$; field of view, $400 \mathrm{~mm}$; flip angle, 150; slice thickness, $5 \mathrm{~mm}$ ) on axial and coronal planes. The bronchial and parenchymal abnormalities were compared with those identified by CT scan applying a modified Bhalla scoring system to assess bronchiectasis, bronchial wall thickening, number of bronchial generations involved, mucous plugging, consolidations, emphysema, bullae, and nodules.

Results: CT scan and MRI findings were comparable for moderate to severe degrees of bronchial and parenchymal alterations. A low concordance was found between MRI and CT scan for lower scores of bronchial abnormalities. CT scan allowed a better identification of peripheral airways abnormalities.

Conclusions: Lung alterations in patients with higher radiation sensitivity, such as patients with CVID, might be evaluated by MRI, a radiation-free technique alternative to CT scan.
\end{abstract}

CHEST 2011; 140(6):1581-1589

Abbreviations: BLADE = rotating blade-like k-space covering; CVID = common variable immunodeficiency

$\mathrm{C}$ ommon variable immunodeficiency (CVID) is the

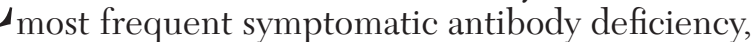
characterized by low levels of serum immunoglobulins and impaired antibody response. Respiratory tract

Manuscript received December 7, 2010; revision accepted April 26, 2011.

Affiliations: From the Department of Radiological Sciences (Drs Serra, Catalano, and Fraioli) and the Department of Molecular Medicine (Drs Milito, Mitrevski, Granata, Martini, Pesce, Sfika, Bonanni, and Quinti), Sapienza University of Rome, Rome, Italy. Funding/Support: This work was supported by the European Commission [Grant HEALTH-F2-2008-201549].

Correspondence to: Isabella Quinti, MD, PhD, Department of Molecular Medicine, Sapienza University of Rome, Fondazione Eleonora Lorillard Spencer Cenci, Rome, Italy; e-mail: isabella. quinti@uniromal.it

(C) 2011 American College of Chest Physicians. Reproduction of this article is prohibited without written permission from the American College of Chest Physicians (http://www.chestpubs.org/ site/misc/reprints.xhtml).

DOI: $10.1378 /$ chest.10-3147 infections are the most prominent clinical problem observed at diagnosis and during follow-up. Despite IgG replacement, patients with CVID still suffer from respiratory infections leading, over time, to permanent lung damage in a high percentage of patients. ${ }^{1}$ The most common lung alterations reported in patients with CVID are represented by bronchiectasis, bronchial wall thickening, nodules, and parenchymal opacities. $^{2}$ Moreover, CVID-associated manifestations can include granulomatous disease and lymphoproliferative disorders. ${ }^{3}$ For these reasons, the management of patients with CVID often entails frequent radiologic examinations, such as chest CT scans.

Although radiation exposure might not be a contraindication in other patient populations with the application of low-dose chest CT scan protocols, ${ }^{4,5}$ patients with CVID may have a fragile somatic profile, determining higher radiation sensitivity. ${ }^{6-8}$ As the exact 
Table 1-Clinical and Immunologic Data and Pulmonary Function Tests of 21 Patients With CVID

\begin{tabular}{|c|c|c|c|c|c|c|c|c|c|c|}
\hline Patient & Age, y & Sex & $\begin{array}{l}\text { Onset } \\
\text { Age, y }\end{array}$ & $\begin{array}{c}\text { Trough IgG, } \\
\text { mg/dL }\end{array}$ & $\begin{array}{c}\mathrm{IgA}, \\
\mathrm{mg} / \mathrm{dL}\end{array}$ & $\begin{array}{l}\text { IgM, } \\
\mathrm{mg} / \mathrm{dL}\end{array}$ & $\mathrm{FEV}_{1}(\%)$ & $\mathrm{FVC}(\%)$ & $\mathrm{FEV}_{\mathrm{l}} / \mathrm{FVC}$ & $\mathrm{MEF}_{25}(\%)$ \\
\hline 1 & 30 & M & 2 & 694.5 & 35 & 23 & $3.43(81)$ & $4.76(96)$ & 0.72 & $4.07(49)$ \\
\hline 2 & 31 & M & 2 & 833.5 & 0 & 0 & $1.93(48.0)$ & $2.87(60.1)$ & 0.67 & $2.37(11.7)$ \\
\hline 3 & 43 & M & 19 & 680.0 & 0 & 0 & $1.61(45.1)$ & $2.48(57.2)$ & 0.64 & $1.98(18.7)$ \\
\hline 4 & 20 & $\mathrm{~F}$ & 8 & 583.0 & 0 & 0 & $2.45(83.2)$ & $2.71(80.4)$ & 0.9 & $2.22(80.7)$ \\
\hline 5 & 58 & M & 25 & 758.0 & 32 & 32 & $2.23(78.6)$ & $3.29(92.9)$ & 0.67 & $1.41(41.8)$ \\
\hline 6 & 60 & $\mathrm{~F}$ & 13 & 835.0 & 6 & 4 & $1.00(54.2)$ & $1.25(56.5)$ & 0.8 & $1.21(36.3)$ \\
\hline 7 & 15 & M & 2 & 678.33 & 0 & 0 & $4.61(120.8)$ & $5.25(117.6)$ & 0.87 & $2.56(114.6)$ \\
\hline 8 & 62 & M & 33 & 671.0 & 0 & 4 & $1.61(50.5)$ & $2.69(66.6)$ & 0.59 & $1.59(26.6)$ \\
\hline 9 & 41 & $\mathrm{M}$ & 22 & 544.0 & 6 & 4 & $3.58(81.5)$ & $5.27(97.7)$ & 0.67 & $2.5(31)$ \\
\hline 10 & 49 & $\mathrm{M}$ & 8 & 615.5 & 6 & 5 & $3.71(98.3)$ & $5.27(113)$ & 0.7 & $2.06(55.3)$ \\
\hline 11 & 32 & M & 13 & 569.5 & 7 & 4 & $2.76(70.9)$ & $4.16(90.1)$ & 0.66 & $2.27(35.5)$ \\
\hline 12 & 69 & $\mathrm{~F}$ & 50 & 943.0 & 0 & 14 & $1.21(54.2)$ & $1.82(68)$ & 0.66 & $1.13(22.8)$ \\
\hline 13 & 32 & M & 3 & 803.0 & 0 & 0 & $3.28(83.5)$ & $5.41(116.5)$ & 0.6 & $2.29(25.1)$ \\
\hline 14 & 46 & M & 36 & 713.0 & 6 & 6 & $2.32(75.2)$ & $2.63(70.9)$ & 0.88 & $1.67(76.9)$ \\
\hline 15 & 39 & M & 24 & 309 & 19 & 25 & $2.69(65.6)$ & $4.53(91)$ & 0.59 & $0.37(15.6)$ \\
\hline 16 & 39 & $\mathrm{~F}$ & 24 & 1,000 & 0 & 17 & $1.44(50)$ & $1.77(53)$ & 0.81 & $1.19(32)$ \\
\hline 17 & 46 & M & 36 & 824 & 0 & 0 & $3.87(118.8)$ & $4.44(112.8)$ & 0.87 & 2.47 (139.6) \\
\hline 18 & 32 & M & 30 & 479 & 0 & 17 & $5.05(109.8)$ & $6.03(108.9)$ & 0.83 & $2.87(106)$ \\
\hline 19 & 42 & $\mathrm{M}$ & 37 & 653 & 0 & 7 & $4.97(122.0 .3)$ & $6.2(124.9)$ & 0.80 & $2.59(112.8)$ \\
\hline 20 & 44 & $\mathrm{M}$ & 20 & 572 & 0 & 0 & $2.02(54.7)$ & $2.86(63.9)$ & 0.70 & $0.76(36.7)$ \\
\hline 21 & 38 & M & 35 & 588 & 9 & 68 & $4.08(99.7)$ & $5.02(101.4)$ & 0.81 & $1.91(81.3)$ \\
\hline
\end{tabular}

The CVID diagnosis was based on the European Society for Immunodeficiencies/Pan-American Group for Immunodeficiency definition..$^{15}$ Trough IgG

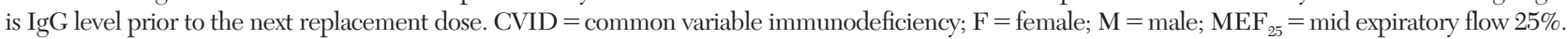

level of "safe" radiation exposure is unknown, clinicians should consider a risk-benefit assessment when ordering CT scans. The validation of a safe, noninvasive, radiation-free technique is needed.

MRI of the lung has been considered experimental for many years, because of the weak signal intensity derived from lung parenchyma, leading to poor image quality. With the development of faster MRI techniques (parallel image acquisition methods), highergradient field strengths, matrix coils, and dedicated free respiratory sequences, high-quality examinations have become available in clinical practice ${ }^{9}$; therefore, the use of lung MRI has been demonstrated to be a reliable

\section{Table 2-Patients With CVID With Bronchial and Parenchymal Abnormalities Identified by CT Scan and MRI}

\begin{tabular}{lrr}
\hline \hline Abnormality & CT Scan & MRI \\
\hline Bronchial abnormalities & & \\
$\quad$ Bronchial wall thickening & $12(57.1)$ & $10(47.6)$ \\
Bronchiectasis & $11(52.4)$ & $9(42.8)$ \\
Mucus plugging & $9(42.8)$ & $8(38.1)$ \\
Involvement of bronchial generations & $15(71.4)$ & $11(52.4)$ \\
$\quad$ up to the fifth and distal & & \\
Parenchymal abnormalities & & $7(33.3)$ \\
$\quad$ Emphysema & $12(57.1)$ & $10(47.6)$ \\
$\quad$ Nodules & $8(38.1)$ & $8(38.1)$ \\
Consolidation & $1(4.8)$ & $1(4.8)$ \\
Abscesses & $1(4.8)$ & 0 \\
$\quad$ Bullae & & \\
\hline
\end{tabular}

Data are presented as No. (\%). See Table 1 legend for expansion of abbreviation. imaging method in patients with COPD, cystic fibrosis, and other lung diseases. ${ }^{10-13}$ We have developed a dedicated MRI protocol to compare the accuracy of morphologic MRI and chest CT scan in the evaluation of lung alterations in a cohort of 21 patients with CVID.

\section{Materials AND Methods}

\section{Patient Population}

Between May 2009 and January 2011, 21 consecutive patients with CVID ( 17 men, four women, mean age $41 \pm 5$ years, range 15-69 years) were included in the study. The diagnosis was based on the European Society for Immunodeficiencies/Pan-American Group for Immunodeficiency definition. ${ }^{14}$ The mean age at diagnosis was $21 \pm 23$ years. All patients were followed up and treated with immunoglobulin replacement therapy according to the Italian national guidelines (www.aieop.org). Relevant clinical and immunologic data and pulmonary function tests at the time of the study are described in Table $1 .{ }^{15}$ All subjects were evaluated with a low-dose chest CT scan and with MRI study on the same day. Two patients were mild smokers. All participants provided written informed consent, and the study was approved by the institutional review board at Sapienza University of Rome.

\section{MRI Protocol}

Chest MRI studies were performed on a 1.5 Tesla imager (Magnetom Avanto; Siemens; Enlargen, Germany) and included a respiratory-triggered $\mathrm{T} 2$-weighted rotating blade-like $\mathrm{k}$-space covering (BLADE) sequence (time repetition, 2,000; echo train $=27$; field of view, $400 \mathrm{~mm}$; flip angle, 150; slice thickness: $5 \mathrm{~mm}$ ) acquired on axial and coronal planes. The technical features of this sequence have been described in detail in previous studies. ${ }^{15,16}$ 
Scan time depended on the respiratory status and size of the patient, ranging between 7 and 16 min (mean, $13 \mathrm{~min}$ ).

\section{CT Examination}

CT images were acquired with a 64-detector row spiral CT scanner (Volume Sensation Cardiac; Siemens), without injection of IV contrast agent, from the lung apices to the upper abdomen in a single breath-hold at the end of full inspiration. The parameters used for acquisition were as follows: $100 \mathrm{kV}$, CAREdose (Siemens) with quality reference set at $100 \mathrm{mAs}$; collimation, $64 \times 0.6 \mathrm{~mm}$; gantry rotation time, $0.33 \mathrm{~s}$; scan time, $6 \mathrm{~s}$; reconstruction kernel, B30 (for mediastinal evaluation) and B60 (for lung parenchyma evaluation). Dose length product (calculated in $\mathrm{mGy} \times \mathrm{cm}$ ) and effective dose (calculated in $\mathrm{mSv}$ ) were recorded for each examination.

\section{Evaluation of Lung Abnormalities on MRI and CT Scan}

Anonymous MRI and CT scan studies were scored in a random order by two independent observers in consensus, adopting a previously validated CT scan scoring system for lung alterations (Bhalla scoring system ${ }^{17}$; type, severity, and extent of nine radiologic findings were assessed: five related to bronchial pathology (bronchiectasis extension, bronchial wall thickening, bronchiec-

\section{Bronchial wall thickening}
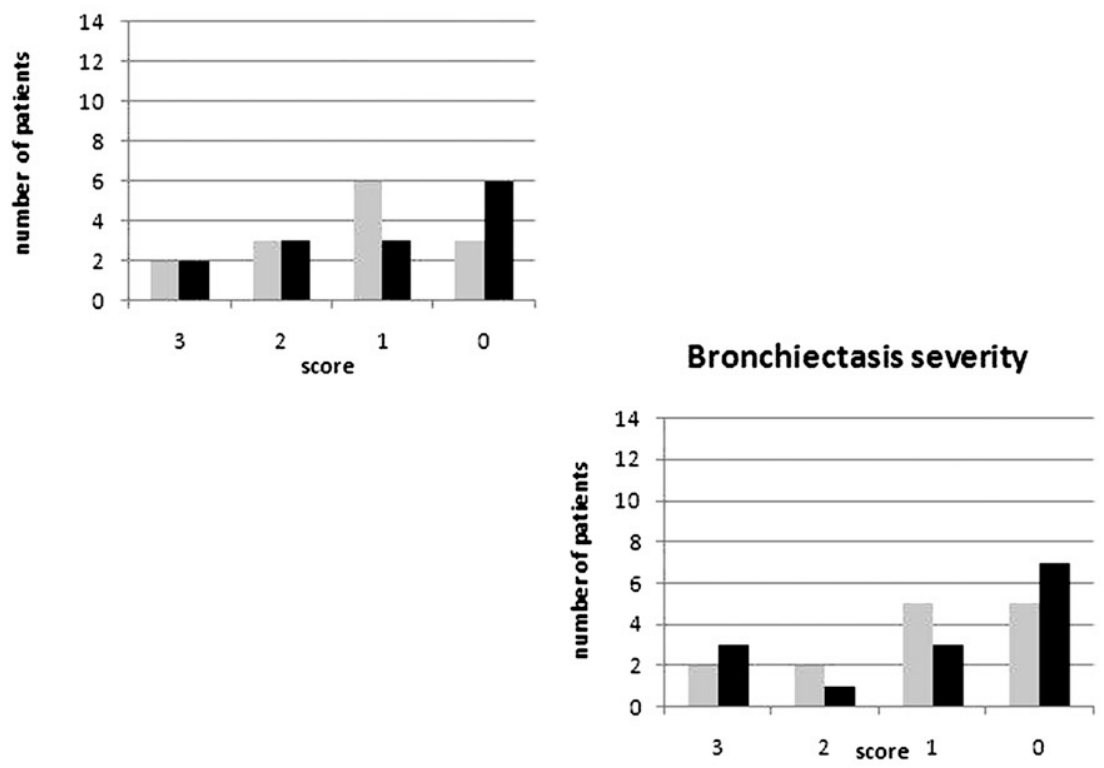

Mucus plugging

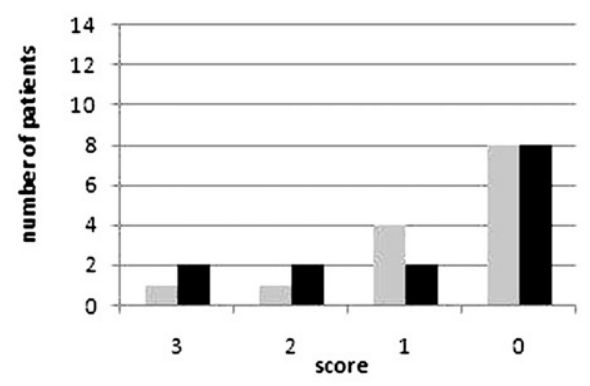

\section{Bronchiectasis severity}

tasis severity, mucus plugging, bronchial generations involved) and four related to parenchymal abnormalities (sacculations or abscesses, consolidations, bullae, emphysema). An extra category was introduced for the assessment of nodules. Nodules were scored according to their number (score $=0$, no nodules detected; score $=1,<5$ nodules detected; score $=2,>5$ nodules detected) and size: $($ score $=1,<1 \mathrm{~cm}$; score $=2,>1 \mathrm{~cm})$. Dimension of nodules was scored as the most common nodule dimension detected on both lungs (e-Table 1).

\section{Statistical Analysis}

Parametric and nonparametric data are presented as mean \pm SD or range, as indicated. For comparison of changes between methods, the Student $t$ test and Mann-Whitney test were used for parametric or nonparametric data sets. A $P$ value of $<.05$ was taken as the threshold of statistical significance.

\section{RESULTS}

\section{CT Scan and MRI Acquisition}

All CT scan and MRI studies were acquired with no complications for all patients. Mean room time for
Bronchiectasis extension

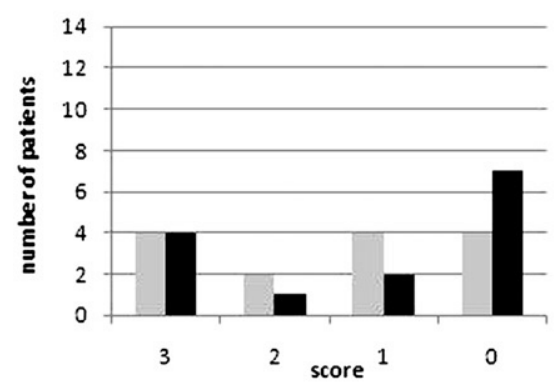

Bronchial generation involved

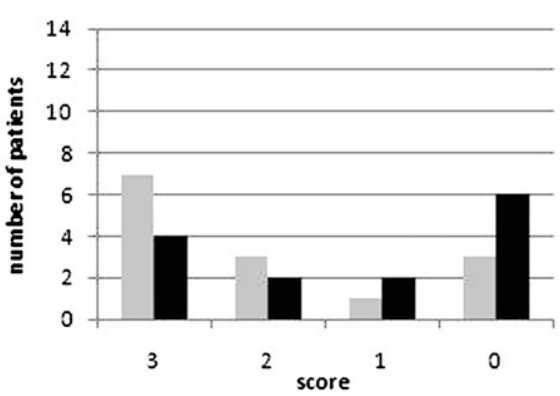

FIGURE 1. Number of patients with common variable immunodeficiency with a defined bronchial abnormality score (bronchiectasis extension, bronchial wall thickening, bronchiectasis severity, mucus plugging, number of bronchial generations involved) identified by CT scan and MRI. Gray bars refer to MRI data; black bars refer to CT scan data. 
CT scan was $4 \pm 2$ min. Average \pm SD dose length product was $106 \pm 21 \mathrm{mGy} \times \mathrm{cm}$; effective dose was $1.5 \pm 0.5 \mathrm{mSv}$. Mean room time for MRI study was $21 \pm 3 \mathrm{~min}$.

\section{MRI and CT Scan in the Evaluation of Lung Abnormalities}

MRI was compared with chest CT scan on a cohort of 21 patients with CVID for the identification of morphologic abnormalities. We adopted a previously validated CT scan scoring system, ${ }^{17}$ adding an extra category represented by nodules (scored by number and dimensions). Overall, the evaluation of lung abnormalities demonstrated that $71 \%$ of patients had bronchial and/or parenchymal abnormalities identified by CT scan and MRI; $23 \%$ of patients had bronchial and/or parenchymal abnormalities identified by CT scan only, and two patients had no lung alterations.

\section{Bronchial Abnormalities}

The most common lung alterations already reported in patients with CVID were bronchial abnormalities. ${ }^{2}$ We confirmed that bronchial wall thickening, bronchiectasis, and mucus plugging were frequent findings (Table 2). These abnormalities were scored according to their severity. We had comparable results between CT scan and MRI in detecting bron- chiectasis severity and extension in those patients who had high and moderate grade of bronchial pathology (Fig 1). MRI performance was weaker in detecting mild abnormalities, identified in five patients by CT scan only. A low concordance was found between MRI and CT scan in the assessment of the number of bronchial generations involved: CT scan scores demonstrated a better identification of peripheral airways abnormalities, reporting an involvement of bronchial generations up to the fifth and distal (scores 2 and 3 ) in $66.6 \%$ of the cases. These abnormalities were identified in $38.1 \%$ of patients by MRI (Tables 2, 3).

\section{Parenchymal Abnormalities}

Parenchymal abnormalities were identified and scored according to their extension. CT scan and MRI gave similar results in detecting the presence and extension of consolidation, abscesses, and emphysema, with overlapping degrees of severity (Fig 2, Tables 2, 4). Abscesses and bullae were rarely reported. The extra category represented by nodules was relevant in our evaluation: Nodules were reported in 12 patients (57.1\%) by CT scan and 10 patients (47.6\%) by MRI. Nodule dimension was similarly scored with the exception of the two patients who had nodules $<1 \mathrm{~cm}$ identified by CT scan only. Nodules presented a maximum transversal diameter ranging between 3 and $17 \mathrm{~mm}$. Figure 3 shows CT scan and MRI images of peripheral bronchial wall thickening;

Table 3-Bronchial Abnormalities

\begin{tabular}{|c|c|c|c|c|c|c|c|c|c|c|}
\hline \multirow[b]{2}{*}{ Patient } & \multicolumn{2}{|c|}{$\begin{array}{l}\text { Bronchial Wall } \\
\text { Thickening }\end{array}$} & \multicolumn{2}{|c|}{$\begin{array}{c}\text { Bronchiectasis } \\
\text { Extension }\end{array}$} & \multicolumn{2}{|c|}{$\begin{array}{c}\text { Bronchiectasis } \\
\text { Severity }\end{array}$} & \multicolumn{2}{|c|}{$\begin{array}{l}\text { Mucus } \\
\text { Plugging }\end{array}$} & \multicolumn{2}{|c|}{$\begin{array}{c}\text { Bronchial Generation } \\
\text { Involved }\end{array}$} \\
\hline & CT Scan & MRI & CT Scan & MRI & CT Scan & MRI & CT Scan & MRI & CT Scan & MRI \\
\hline 1 & 0 & 0 & 0 & 0 & 0 & 0 & 0 & 0 & 0 & 0 \\
\hline 2 & 3 & 3 & 2 & 2 & 3 & 3 & 1 & 2 & 3 & 3 \\
\hline 3 & 2 & 2 & 3 & 3 & 1 & 2 & 1 & 2 & 2 & 2 \\
\hline 4 & 1 & 0 & 1 & 1 & 1 & 1 & 0 & 0 & 2 & 1 \\
\hline 5 & 1 & 1 & 1 & 1 & 2 & 3 & 2 & 2 & 2 & 1 \\
\hline 6 & 2 & 2 & 3 & 3 & 1 & 1 & 0 & 0 & 3 & 3 \\
\hline 7 & 0 & 0 & 1 & 0 & 1 & 0 & 0 & 0 & 3 & 0 \\
\hline 8 & 1 & 0 & 1 & 0 & 0 & 0 & 0 & 0 & 3 & 0 \\
\hline 9 & 3 & 3 & 3 & 3 & 3 & 3 & 3 & 3 & 3 & 3 \\
\hline 10 & 1 & 1 & 1 & 0 & 1 & 0 & 0 & 0 & 1 & 0 \\
\hline 11 & 0 & 0 & 0 & 0 & 0 & 0 & 0 & 0 & 0 & 0 \\
\hline 12 & 1 & 1 & 0 & 0 & 0 & 0 & 1 & 1 & 2 & 2 \\
\hline 13 & 2 & 2 & 3 & 3 & 2 & 1 & 1 & 1 & 3 & 3 \\
\hline 14 & 1 & 1 & 2 & 1 & 1 & 1 & 0 & 0 & 3 & 1 \\
\hline 15 & 0 & 0 & 0 & 0 & 0 & 0 & 0 & 0 & 0 & 0 \\
\hline 16 & 1 & 1 & 2 & 2 & 3 & 3 & 2 & 1 & 3 & 3 \\
\hline 17 & 0 & 0 & 0 & 0 & 0 & 0 & 0 & 0 & 0 & 0 \\
\hline 18 & 0 & 0 & 0 & 0 & 0 & 0 & 0 & 0 & 0 & 0 \\
\hline 19 & 0 & 0 & 0 & 0 & 0 & 0 & 0 & 0 & 0 & 0 \\
\hline 20 & 0 & 0 & 0 & 0 & 0 & 0 & 1 & 0 & 3 & 0 \\
\hline 21 & 0 & 0 & 0 & 0 & 0 & 0 & 1 & 1 & 2 & 2 \\
\hline
\end{tabular}

Morphologic MRI and CT scan studies scored in a random order by two independent observers in consensus, adopting a validated CT scan scoring system for lung alterations (Bhalla scoring system). ${ }^{17}$ 
CT scan and MRI images of diffuse lung disease with bronchiectasis, bronchial wall thickening, and peripheral nodule; and CT scan and MRI images of bronchiectasis and mucus plugging localized in the right upper region in three patients with CVID.

\section{DISCUSSION}

Frequent pulmonary infections are the main CVIDassociated comorbidities leading over time to chronic lung disease. ${ }^{1}$ Bronchial airways and parenchyma are affected with a wide spectrum of permanent alter-

Consolidation

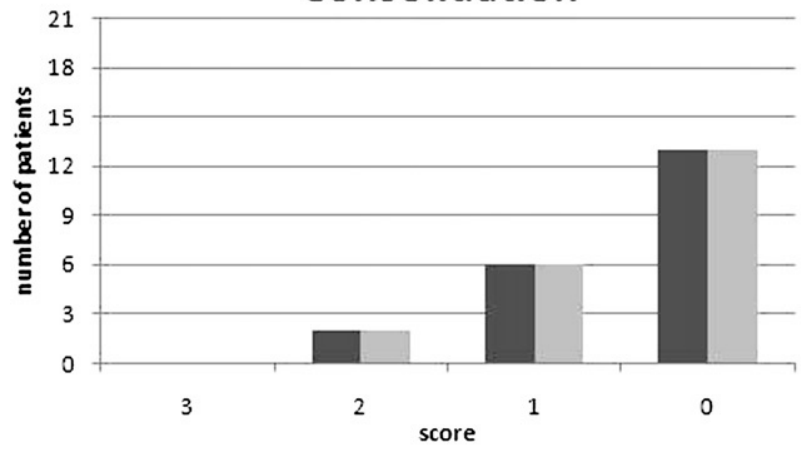

Bullae

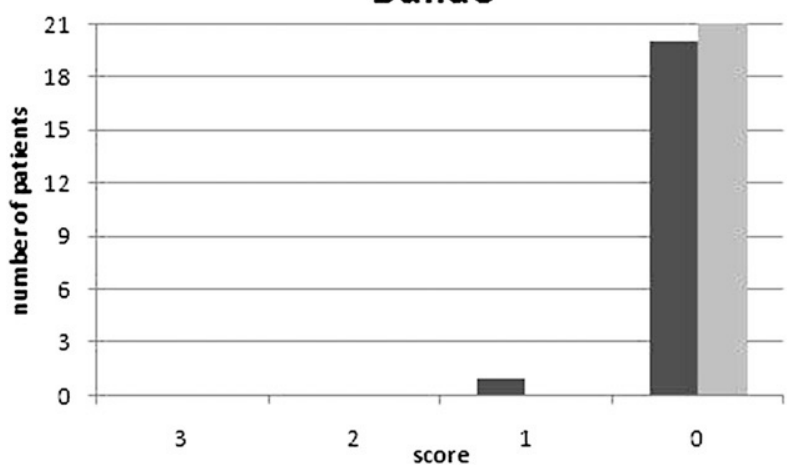

Emphysema

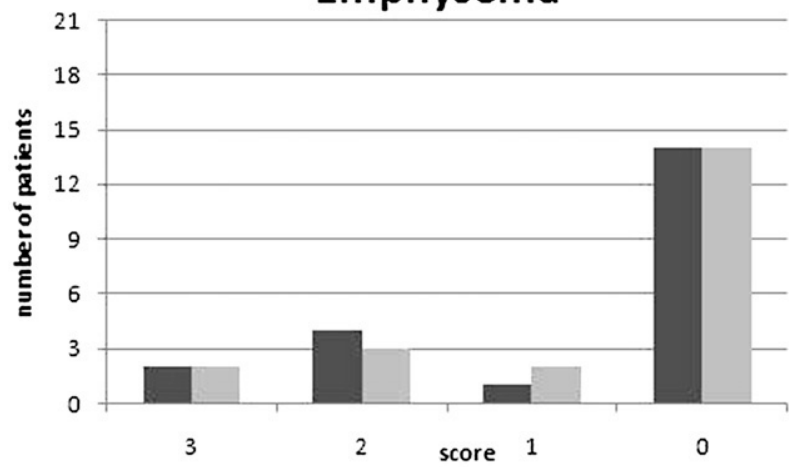

ations, which can show different degrees of severity. ${ }^{2}$ We have recently demonstrated in a large multicenter prospective cohort study that despite immunoglobulin replacement therapy, pneumonia episodes still occur with an estimated incidence of 0.10 episodes/patient/y. Of more concern is the progression of lung disease occurring in about one-half of patients with CVID. Bronchiectasis was the major risk factor identified for pneumonia. ${ }^{18}$ Moreover, the analysis of BAL samples of patients with CVID with bronchiectasis showed that the ongoing pulmonary damage may also be due to subclinical infections. ${ }^{19}$ Therefore, repeated imaging assessments over time by chest CT scan are required
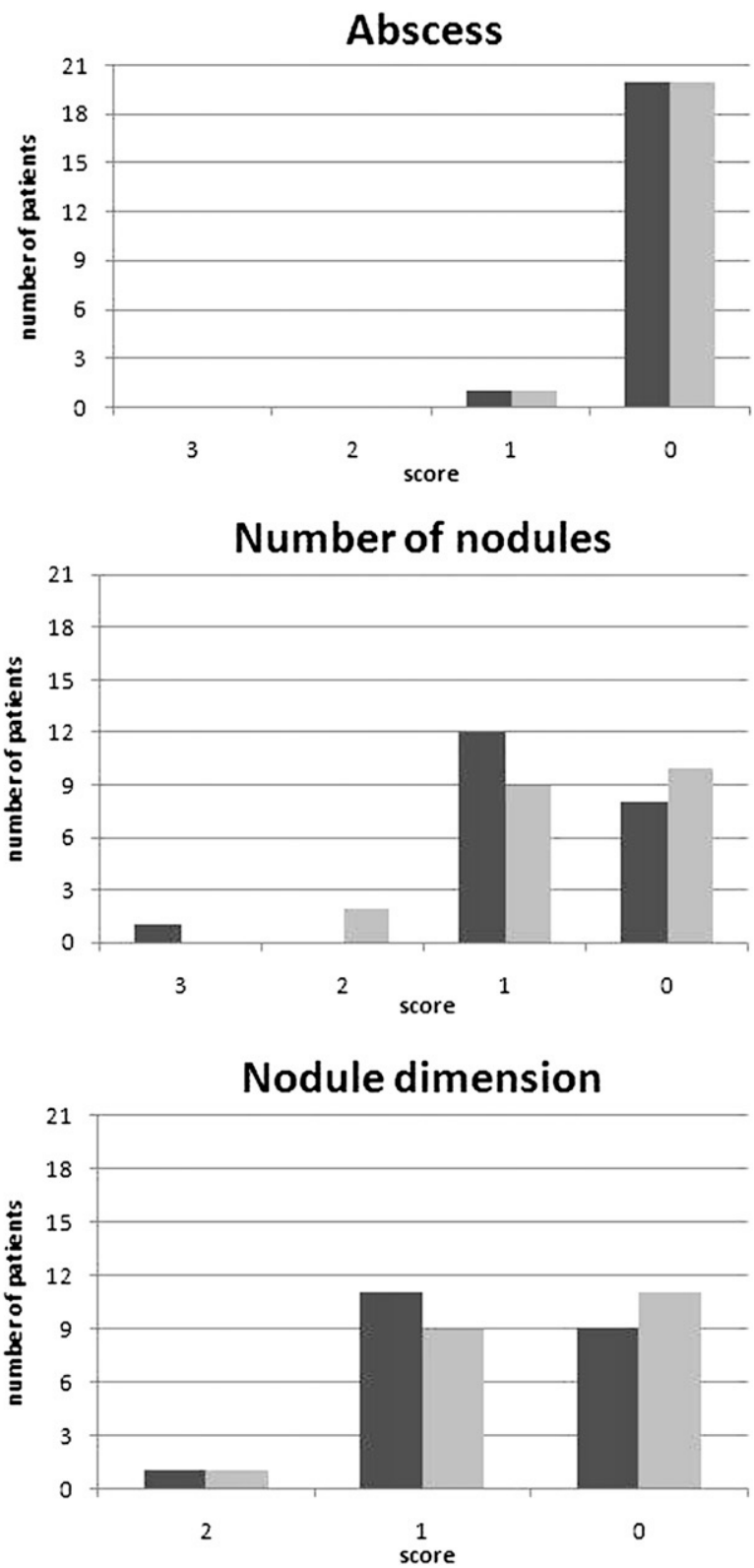

FIGURE 2. Number of patients with common variable immunodeficiency with a defined parenchymal abnormality score (abscesses, consolidations, bullae, emphysema, nodules number, and dimension) identified by CT scan and MRI. Gray bars refer to MRI data; black bars refer to CT scan data. 
Table 4-Parenchymal Abnormalities

\begin{tabular}{|c|c|c|c|c|c|c|c|c|c|c|c|c|}
\hline \multirow[b]{2}{*}{ Patient } & \multicolumn{2}{|c|}{ Emphysema } & \multicolumn{2}{|c|}{ Consolidations } & \multicolumn{2}{|c|}{ Abscesses } & \multicolumn{2}{|c|}{ Bullae } & \multicolumn{2}{|c|}{$\begin{array}{c}\text { Number of } \\
\text { Nodules }\end{array}$} & \multicolumn{2}{|c|}{$\begin{array}{c}\text { Nodules } \\
\text { Dimension }\end{array}$} \\
\hline & CT Scan & MRI & CT Scan & MRI & CT Scan & MRI & CT Scan & MRI & CT Scan & MRI & CT Scan & MRI \\
\hline 1 & 0 & 0 & 0 & 0 & 0 & 0 & 0 & 0 & 0 & 0 & 0 & 0 \\
\hline 2 & 2 & 2 & 2 & 2 & 0 & 0 & 0 & 0 & 1 & 1 & 1 & 1 \\
\hline 3 & 1 & 1 & 1 & 1 & 1 & 1 & 0 & 0 & 1 & 2 & 1 & 1 \\
\hline 4 & 0 & 0 & 0 & 0 & 0 & 0 & 0 & 0 & 1 & 1 & 0 & 0 \\
\hline 5 & 2 & 2 & 0 & 0 & 0 & 0 & 0 & 0 & 0 & 0 & 0 & 0 \\
\hline 6 & 2 & 2 & 0 & 0 & 0 & 0 & 0 & 0 & 1 & 1 & 1 & 1 \\
\hline 7 & 0 & 0 & 0 & 0 & 0 & 0 & 0 & 0 & 0 & 0 & 0 & 0 \\
\hline 8 & 3 & 3 & 0 & 0 & 0 & 0 & 0 & 0 & 1 & 1 & 1 & 1 \\
\hline 9 & 3 & 3 & 0 & 0 & 0 & 0 & 0 & 0 & 1 & 1 & 1 & 1 \\
\hline 10 & 0 & 0 & 0 & 0 & 0 & 0 & 0 & 0 & 1 & 1 & 1 & 1 \\
\hline 11 & 0 & 0 & 0 & 0 & 0 & 0 & 0 & 0 & 0 & 0 & 0 & 0 \\
\hline 12 & 0 & 0 & 0 & 0 & 0 & 0 & 0 & 0 & 3 & 2 & 2 & 2 \\
\hline 13 & 2 & 1 & 2 & 2 & 0 & 0 & 0 & 0 & 0 & 0 & 0 & 0 \\
\hline 14 & 0 & 0 & 0 & 0 & 0 & 0 & 0 & 0 & 0 & 0 & 0 & 0 \\
\hline 15 & 0 & 0 & 1 & 1 & 0 & 0 & 0 & 0 & 1 & 1 & 1 & 1 \\
\hline 16 & 0 & 0 & 1 & 1 & 0 & 0 & 0 & 1 & 1 & 1 & 1 & 1 \\
\hline 17 & 0 & 0 & 1 & 1 & 0 & 0 & 0 & 0 & 0 & 0 & 0 & 0 \\
\hline 18 & 0 & 0 & 0 & 0 & 0 & 0 & 0 & 0 & 1 & 0 & 1 & 0 \\
\hline 19 & 0 & 0 & 1 & 1 & 0 & 0 & 0 & 0 & 0 & 0 & 0 & 0 \\
\hline 20 & 0 & 0 & 1 & 1 & 0 & 0 & 0 & 0 & 1 & 0 & 1 & 0 \\
\hline 21 & 0 & 0 & 0 & 0 & 0 & 0 & 0 & 0 & 1 & 1 & 1 & 1 \\
\hline
\end{tabular}

Morphologic MRI and CT scan studies scored in a random order by two independent observers in consensus, adopting a validated CT scan scoring system for lung alterations (Bhalla scoring system). ${ }^{17}$ The extra category represented by nodules (number and dimension) has been added to the scoring system.

for the diagnosis of acute and chronic respiratory comorbidities. However, an increased radiosensitivity has been described in patients affected by many primary immunodeficiencies, such as ataxia teleangiecta$\mathrm{sia}^{20}$ and CVID. These patients have a increased risk of lymphoproliferative diseases and gastric cancer ${ }^{1,21,22}$ and a higher incidence of chromosomal aberrations after x-ray irradiation compared with healthy control subjects. ${ }^{6-8}$ Moreover, some primary immunodeficiencies, including CVID, have been explained by defects in DNA repair pathways. ${ }^{23}$ Therefore, patients with CVID should be protected from diagnostic and therapeutic procedures using ionizing radiation. Chest MRI was proposed as a potential alternative for the study of the lungs in the late 1980s. ${ }^{24}$ Even though spatial resolution is still lower than CT scan, lung MRI is totally radiation-free and also offers the advantages of better tissue characterization and the possibility of performing functional studies..$^{25}$

Similarly to other published studies, ${ }^{10-13}$ we analyzed the possibility of comparing MRI to chest CT scan findings in a cohort of patients with CVID. We adopted a previously validated CT scan scoring system, ${ }^{17}$ adding an extra category represented by nodules. The need for a validated CT scan scoring system to grade lung abnormalities in patients affected by primary immunodeficiencies has been recently outlined. ${ }^{26,27}$ We reported comparable results in the scoring of bronchial and parenchymal abnormalities between morphologic CT scan and MRI. The concordance between the two techniques was good in patients who had severe and moderate degrees of defects. However, MRI was less sensitive in detecting mild bronchial alterations. This was mainly due to the low MRI sensitivity in the assessment of peripheral bronchial alterations, which can be explained by the loss of signal in the peripheral areas of lung parenchyma, as discussed in previous studies performed on other lung diseases, such as cystic fibrosis. ${ }^{28}$

Different MRI protocols have been proposed to evaluate lung parenchyma, ${ }^{29,30}$ with the rationale to obtain a compromise between the rapid decay of signal intensity of lung parenchyma and the susceptibility effects induced by endoalveolar gas; shorter echo times (in gradient-echo sequences) or shorter echo spacing (in turbo spin echo sequences) have been suggested to overcome these technical constrains. More recently, half-Fourier-acquired singleshot turbo spin echo sequences have been proposed by various authors, yielding high-quality images with fewer artifacts. Short echo spacing makes this pulse sequence relatively resistant to the inhomogeneous magnetic susceptibility of lung parenchyma, whereas the short acquisition time compensates respiratory and cardiac motion.

Even if in our institution the standard routine protocol for pulmonary MRI includes several of the previously mentioned sequences ( $\mathrm{T} 2$ half-Fourier-acquired 


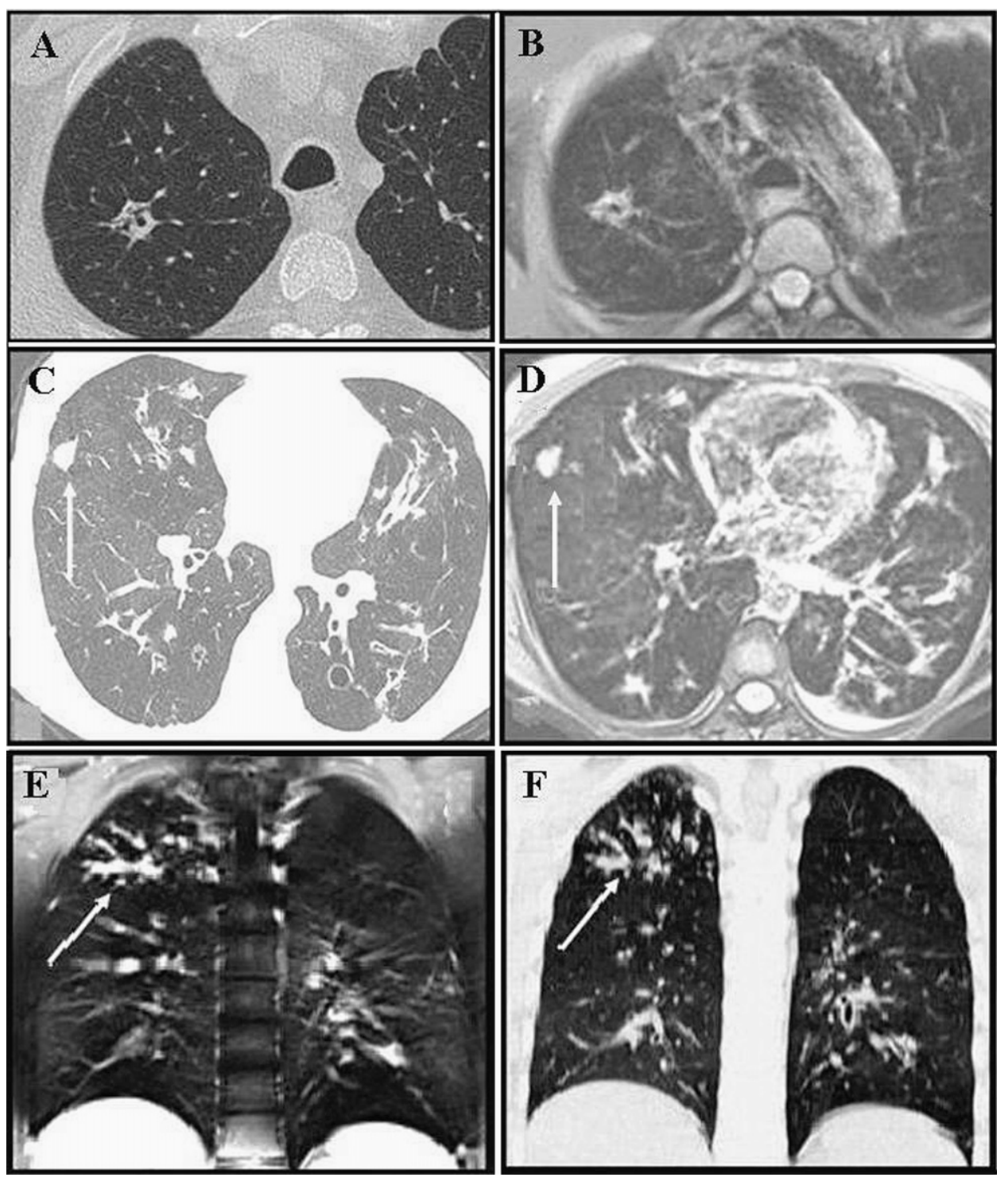

FIGURE 3. A, CT image of peripheral bronchial wall thickening in a patient with common variable immunodeficiency (CVID). B, MRI image of peripheral bronchial wall thickening in a patient with CVID. C, CT image of diffuse lung disease in a patient with bronchiectasis, bronchial wall thickening, and peripheral nodule (arrow). D, MRI image of diffuse lung disease in a patient with bronchiectasis, bronchial wall thickening, and peripheral nodule (arrow). E, MRI image from a patient with bronchiectasis and mucus plugging localized in the right upper region (arrow). F, CT image from a patient with bronchiectasis and mucus plugging localized in the right upper region (arrow).

single-shot turbo spin echo; T1 volume interpolated gradient recalled echo precontrast and postcontrast administration), in our study we chose to use only a dedicated BLADE sequence for morphologic imaging. We needed to select a single morphologic sequence on which to apply our scoring system: a score derived from the assessment of more image data sets-acquired with different parameterswould have affected the reproducibility of the evaluation. No previous studies have been reported on the application of BLADE sequences in the evaluation of the lung. On the basis of previous international studies performed on brain and abdominal imaging and our experience, the BLADE sequence seemed to be most accurate and reproducible for a comprehensive assessment of parenchymal and bronchial pathology, for several reasons: (1) It is a respiratorytriggered sequence showing few breathing artifacts; (2) it has a radial readout of the k-space, which results in much sharper images even if the patient is moving; and (3) it is a slightly longer compared with breathhold imaging, but may allow the avoidance of scan repetition in uncooperative patients. . $^{31,32}$ All MRI studies were acquired with the application of a navigator for respiratory triggering. This method was chosen to achieve a further reduction of movement artifacts 
related to respiratory activity in less cooperative patients who were not able to maintain an adequate apnea with the application of breath-hold imaging. Because of the application of a navigator for respiratory triggering, all MRI studies were acquired in an expiratory phase, whereas all CT scans were obtained at the end of full inspiration; this did not represent an issue, since the assessment of all morphologic alterations included in our scoring system was not influenced by the respiratory phase adopted. Previous studies of cystic fibrosis lung disease reported that the evaluation of inspiratory and expiratory CT scans match closely. ${ }^{33}$

In conclusion, MRI represents a promising radiationfree technique to evaluate lung alterations in patients with higher radiation sensitivity, such as patients with CVID. CT scan remains the gold standard for diagnosing initial changes in the course of bronchial pathology; MRI can have a relevant role in patients with CVID with a moderate or high degree of structural lung abnormalities. Even with the limits of low spatial resolution, high costs, and low availability, we propose MRI as a possible radiation-free alternative to CT scanning in patients with CVID. Further studies on larger groups of patients are needed to improve image quality and for a definitive standardization of the acquisition protocol.

\section{ACKNOWLEDGMENTS}

Author contributions: Dr Serra: contributed to designing the research; analyzing the data; and writing, revision, review, and approval of the manuscript.

Dr Milito: contributed to performing the research; collecting and analyzing the data; and writing, revision, review, and approval of the manuscript.

Dr Mitrevski: contributed to performing the research; collecting and analyzing the data; and writing, revision, review, and approval of the manuscript

Dr Granata: contributed to performing the research; collecting and analyzing the data; and writing, revision, review, and approval of the manuscript.

Dr Martini: contributed to performing the statistical analysis, and writing, revision, review, and approval of the manuscript.

Dr Pesce: contributed to ascertaining patients' clinical and immunological data, and writing, revision, review, and approval of the manuscript.

Dr Sfika: contributed to performing the research; collecting and analyzing the data; and writing, revision, review, and approval of the manuscript.

Dr Bonanni: contributed to the collection of data, writing, revision, review, and approval of the manuscript.

Dr Catalano: contributed to performing the research; collecting and analyzing the data; and writing, revision, review, and approval of the manuscript.

Dr Fraioli: contributed to designing the research; analyzing the data; and writing, revision, review, and approval of the manuscript. Dr Quinti: contributed to designing the research; analyzing the data; and writing, revision, review, and approval of the manuscript. Financial/nonfinancial disclosures: The authors have reported to CHEST that no potential conflicts of interest exist with any companies/organizations whose products or services may be discussed in this article.

Role of sponsors: The sponsor had no role in the design of the study, the collection and analysis of the data, or in the preparation of the manuscript.
Other contributions: We thank all the patients who participated in this study, as well as their families, and the Jeffrey Modell Foundation.

Additional information: The e-Table can be found in the Online Supplement at http://chestjournal.chestpubs.org/content/ 140/6/1581/DC1.

\section{REFERENCES}

1. Quinti I, Soresina A, Spadaro G, et al; Italian Primary Immunodeficiency Network. Long-term follow-up and outcome of a large cohort of patients with common variable immunodeficiency. J Clin Immunol. 2007;27(3):308-316.

2. Tanaka N, Kim JS, Bates CA, et al. Lung diseases in patients with common variable immunodeficiency: chest radiographic, and computed tomographic findings. J Comput Assist Tomogr. 2006;30(5):828-838.

3. Chapel H, Lucas M, Lee M, et al. Common variable immunodeficiency disorders: division into distinct clinical phenotypes. Blood. 2008;112(2):277-286.

4. O'Connor OJ, Vandeleur M, McGarrigle AM, et al. Development of low-dose protocols for thin-section CT assessment of cystic fibrosis in pediatric patients. Radiology. 2010; 257(3):820-829.

5. Kim MJ, Park CH, Choi SJ, Hwang KH, Kim HS. Multidetector computed tomography chest examinations with lowkilovoltage protocols in adults: effect on image quality and radiation dose. J Comput Assist Tomogr. 2009;33(3):416-421.

6. Aghamohammadi A, Moin M, Kouhi A, et al. Chromosomal radiosensitivity in patients with common variable immunodeficiency. Immunobiology. 2008;213(5):447-454.

7. Palanduz S, Palanduz A, Yalcin I, et al. In vitro chromosomal radiosensitivity in common variable immune deficiency. Clin Immunol Immunopathol. 1998;86(2):180-182.

8. Vorechovský I, Scott D, Haeney MR, Webster DA. Chromosomal radiosensitivity in common variable immune deficiency. Mutat Res. 1993;290(2):255-264.

9. Puderbach M, Hintze C, Ley S, Eichinger M, Kauczor HU, Biederer J. MR imaging of the chest: a practical approach at 1.5T. Eur J Radiol. 2007;64(3):345-355.

10. Montella S, Santamaria F, Salvatore M, et al. Assessment of chest high-field magnetic resonance imaging in children and young adults with noncystic fibrosis chronic lung disease: comparison to high-resolution computed tomography and correlation with pulmonary function. Invest Radiol. 2009;44(9): $532-538$

11. Ley-Zaporozhan J, Ley S, Kauczor HU. Morphological and functional imaging in COPD with CT and MRI: present and future. Eur Radiol. 2008;18(3):510-521.

12. Sverzellati N, Molinari F, Pirronti T, Bonomo L, Spagnolo P, Zompatori M. New insights on COPD imaging via CT and MRI. Int J Chron Obstruct Pulmon Dis. 2007;2(3):301-312.

13. Puderbach M, Eichinger M, Haeselbarth J, et al. Assessment of morphological MRI for pulmonary changes in cystic fibrosis $(\mathrm{CF})$ patients: comparison to thin-section $\mathrm{CT}$ and chest x-ray. Invest Radiol. 2007;42(10):715-725.

14. Conley ME, Notarangelo LD, Etzioni A; Representing PAGID (Pan-American Group for Immunodeficiency) and ESID (European Society for Immunodeficiencies). Diagnostic criteria for primary immunodeficiencies. Clin Immunol. 1999; 93(3):190-197.

15. Bayramoglu S, Kilickesmez O, Cimilli T, et al. T2-weighted MRI of the upper abdomen: comparison of four fat-suppressed T2-weighted sequences including PROPELLER (BLADE) technique. Acad Radiol. 2010;17(3):368-374

16. Fujimoto K, Koyama T, Tamai K, Morisawa N, Okada T, Togashi K. BLADE acquisition method improves T2-weighted 
MR images of the female pelvis compared with a standard fast spin-echo sequence [published online ahead of print September 8, 2010]. Eur J Radiol. doi:10.1016/j.ejrad. 2010.08.002.

17. Bhalla M, Turcios N, Aponte V, et al. Cystic fibrosis: scoring system with thin-section CT. Radiology. 1991;179(3): 783-788.

18. Quinti I, Soresina A, Guerra A, et al. Effectiveness of immunoglobulin replacement therapy on clinical outcome in patients with primary antibody deficiencies: results from a multicenter prospective cohort study. J Clin Immunol. 2011;31(3):315-322.

19. Cunningham-Rundles C. Lung disease, antibodies and other unresolved issues in immune globulin therapy for antibody deficiency. Clin Exp Immunol. 2009;157(suppl 1):12-16.

20. McKinnon PJ. ATM and ataxia telangiectasia. EMBO Rep. 2004;5(8):772-776.

21. Cunningham-Rundles C, Bodian C. Common variable immunodeficiency: clinical and immunological features of 248 patients. Clin Immunol. 1999;92(1):34-48.

22. Park MA, Li JT, Hagan JB, Maddox DE, Abraham RS. Common variable immunodeficiency: a new look at an old disease. Lancet. 2008;372(9637):489-502.

23. Offer SM, Pan-Hammarström Q, Hammarström L, Harris RS. Unique DNA repair gene variations and potential associations with the primary antibody deficiency syndromes IgAD and CVID. PLoS ONE. 2010;5(8):e12260.

24. Cutillo AG, Morris AH, Ailion DC, Durney CH. Clinical implications of nuclear magnetic resonance lung research. Chest. 1989;96(3):643-652.

25. Mayo JR. MR imaging of pulmonary parenchyma. Magn Reson Imaging Clin N Am. 2000;8(1):105-123.

26. Bondioni MP, Soresina A, Lougaris V, Gatta D, Plebani A, Maroldi R. Common variable immunodeficiency: computed tomography evaluation of bronchopulmonary changes including nodular lesions in 40 patients. Correlation with clinical and immunological data. J Comput Assist Tomogr. 2010;34(3): 395-401.

27. van de Ven AA, van Montfrans JM, Terheggen-Lagro SW, et al. A CT scan score for the assessment of lung disease in children with common variable immunodeficiency disorders. Chest. 2010;138(2):371-379.

28. Failo R, Wielopolski PA, Tiddens HA, Hop WC, Mucelli RP, Lequin MH. Lung morphology assessment using MRI: a robust ultra-short TR/TE 2D steady state free precession sequence used in cystic fibrosis patients. Magn Reson Med. 2009;61(2): 299-306.

29. Hatabu H, Gaa J, Tadamura E, et al. MR imaging of pulmonary parenchyma with a half-Fourier single-shot turbo spinecho (HASTE) sequence. Eur J Radiol. 1999;29(2):152-159.

30. Biederer J, Reuter M, Both M, et al. Analysis of artefacts and detail resolution of lung MRI with breath-hold T1-weighted gradient-echo and T2-weighted fast spin-echo sequences with respiratory triggering. Eur Radiol. 2002;12(2):378-384.

31. Forbes KP, Pipe JG, Karis JP, Farthing V, Heiserman JE. Brain imaging in the unsedated pediatric patient: comparison of periodically rotated overlapping parallel lines with enhanced reconstruction and single-shot fast spin-echo sequences. AJNR Am J Neuroradiol. 2003;24(5):794-798.

32. Rosenkrantz AB, Mannelli L, Mossa D, Babb JS. Breathhold T2-weighted MRI of the liver at 3T using the BLADE technique: impact upon image quality and lesion detection. Clin Radiol. 2011;66(5):426-433.

33. Loeve M, Lequin MH, de Bruijne M, et al. Cystic fibrosis: are volumetric ultra-low-dose expiratory CT scans sufficient for monitoring related lung disease? Radiology. 2009;253(1): 223-229. 